\title{
Correction to: Toward validation of QTLs associated with pod and seed size in common bean using two nested recombinant inbred line populations
}

Ester Murube • Ana Campa • Qijian Song •

Phillip McClean • Juan Jose Ferreira (1)

Published online: 17 July 2020

(C) Springer Nature B.V. 2020

Correction to: Mol Breeding (2020) 40: 7

https://doi.org/10.1007/s11032-019-1085-1

Unfortunately, in the final version of this article the original Table 6 was not included in the proofing step and the Table 5 was duplicated.

The online version of the original article can be found at https://doi. org/10.1007/s11032-019-1085-1

E. Murube · A. Campa · J. J. Ferreira $(\bowtie)$

Plant Genetic Group, Area of Horticultural and Forest Crops, SERIDA, 33300 Villaviciosa, Asturias, Spain

e-mail: jjferreira@serida.org

E. Murube

e-mail: emurube@serida.org

A. Campa

e-mail: acampa@serida.org
Q. Song

USDA-ARS, Soybean Genomics and Improvement Lab, Beltsville, MD 20705, USA

e-mail: Qijian.Song@ars.usda.gov

P. McClean

Department of Plant Sciences, North Dakota State University, Fargo, ND 58108, USA

e-mail: phillip.mcclean@ndsu.edu

P. McClean

North Dakota State University, Fargo, ND 58108, USA 
Table 6 QTL with significant epistatic interaction detected in the RIL population XB

\begin{tabular}{|c|c|c|c|c|c|c|c|c|c|c|c|}
\hline Q & LG & $\begin{array}{l}\text { Position } \\
\text { (cM) }\end{array}$ & tart & End & 2 & LG & $\begin{array}{l}\text { Position } \\
\text { (cM) }\end{array}$ & $\mathrm{rt}$ & End & $\mathrm{AA}^{(1)}$ & $h^{2}$ \\
\hline LEATt. & Pv01 & $685-845$ & NP01_348 & NP01_350 & & Pv09 & $41-83$ & SNP09_098 & SNP09_119 & -1.80 & 0.01 \\
\hline $\mathrm{SA} 12^{\mathrm{XB} *}$ & Pv01 & $1305-1375$ & NP01_543 & SNP01_562 & E-SA10 ${ }^{\mathrm{XB}}$ & Pv10 & 00-11 & NP10_15 & SNP10_16 & 2.26 & 0.02 \\
\hline $\mathrm{E}-\mathrm{SA} 2.1^{\mathrm{XB}}$ & $\mathrm{Pv} 02$ & $565-$ & 30 & 33 & E-SA3 $3^{\mathrm{XB}}$ & 03 & & 597 & 5 & 6 & 03 \\
\hline E-SP3.1 $1^{\mathrm{XB}}$ & Pv03 & $337-395$ & SNP03_212 & SNP03_222 & $\mathrm{SP} 11.2^{\mathrm{XB} *}$ & Pv11 & $674-726$ & SNP11_202 & SNP11_219 & 0.65 & 0.01 \\
\hline $\mathrm{SP} 7.2^{\mathrm{XB} *}$ & Pv07 & 849-889 & NP07_316 & SNP07_317 & $\mathrm{SP} 11.2^{\mathrm{XB} *}$ & Pv11 & $674-726$ & P11_202 & 219 & -0.73 & 0.02 \\
\hline$-\mathrm{SP} 2.2^{\mathrm{XB}}$ & Pv02 & $1401-1476$ & NP0 & 317 & E-SP3. $2^{\mathrm{XB}}$ & 3 & 66 & 0 & 7 & 0.42 & .02 \\
\hline E-SP6.1 $1^{\mathrm{XB}}$ & Pv06 & $57-161$ & SNP06_151 & SNP06_160 & E-SP11.1 ${ }^{\mathrm{XB}}$ & Pv11 & $308-409$ & SNP11_092 & SNP11_095 & 0.53 & 0.01 \\
\hline $\mathrm{SL}^{\mathrm{XB} *}$ & Pv01 & $685-735$ & SNP01_348 & SNP01_350 & E-SL6. $2^{\mathrm{XB}}$ & Pv06 & $676-744$ & SNP06_563 & SNP06_582 & 0.23 & 0.02 \\
\hline E-SL3 ${ }^{\mathrm{XB}}$ & Pv03 & $347-395$ & $3 \_212$ & 222 & $\mathrm{SL} 11^{\mathrm{XB} *}$ & Pv11 & $674-726$ & IP11_202 & 219 & 0.15 & .01 \\
\hline SL6. $2^{\mathrm{XB} *}$ & Pv06 & $676-$ & P06_563 & SNP06_582 & SL7. $2^{\mathrm{XB} *}$ & Pv07 & 833-889 & P07_316 & SNP07_317 & 0.14 & .01 \\
\hline SL7. $1^{\mathrm{XB}} *$ & Pv07 & $395-512$ & JP07_175 & SNP07_188 & $\mathrm{SL} 11^{\mathrm{XB} *}$ & v11 & 6 & P11_202 & SNP11_219 & -0.23 & 0.02 \\
\hline E-SL5 ${ }^{\mathrm{XB}}$ & Pv05 & $773-822$ & 228 & 38 & E-SL6. $1^{\mathrm{XB}}$ & 6 & & 83 & 00 & 4 & 0.01 \\
\hline E-SWI3.2 $2^{\mathrm{XB}}$ & Pv03 & $395-464$ & SNP03_270 & SNP03_277 & & Pv08 & 70 & 35 & 048 & -0.12 & 0.04 \\
\hline E-SWI3.3 $3^{\mathrm{XB}}$ & Pv03 & $1031-1084$ & SNP03_556 & SNP03_561 & E-SWI11 ${ }^{\mathrm{XB}}$ & Pv11 & $603-714$ & SNP11_186 & SNP11_202 & 0.14 & 0.04 \\
\hline E-SWI4 ${ }^{\mathrm{XB}}$ & $\mathrm{Pv} 04$ & $1059-1130$ & NP04_107 & 111 & $\mathrm{SWI}^{\mathrm{XB} *}$ & Pv07 & & 64 & 175 & 0.19 & 0.07 \\
\hline $\mathrm{E}-\mathrm{SW} 2^{\mathrm{XB}}$ & Pv02 & $565-775$ & NP02_130 & NP02_133 & $\mathrm{SW} 3^{\mathrm{XB}} *$ & Pv03 & 37 & NP03_122 & SNP03_145 & -0.34 & 0.01 \\
\hline $\mathrm{SW} 3^{\mathrm{XB}} *$ & Pv03 & $245-337$ & NP03_122 & SNP03_145 & $\mathrm{SW} 7.2^{\mathrm{XB} *}$ & Pv07 & 89 & _316 & 7317 & -0.29 & 0.02 \\
\hline $\mathrm{SW} 7.2^{\mathrm{XB} *}$ & Pv07 & 849-889 & SNP07_316 & SNP07_317 & E-SW11 ${ }^{\mathrm{XB}}$ & Pv11 & $684-766$ & 219 & 226 & -0.30 & 0.02 \\
\hline E-SW5.1 ${ }^{\mathrm{XB}}$ & Pv05 & $205-386$ & SNP05_031 & SNP05_033 & E-SW5.2 $2^{\mathrm{XB}}$ & Pv05 & $1116-1228$ & $5 \_290$ & $5 \_297$ & 0.41 & 0.02 \\
\hline E-PP3 ${ }^{\mathrm{XB}}$ & $\mathrm{Pv} 03$ & $500-504$ & SNP03_356 & SNP03_387 & $\mathrm{PP}^{\mathrm{XB}} *$ & Pv09 & $00-27$ & SNP09_023 & SNP09_049 & -0.42 & 0.02 \\
\hline E-PP2 ${ }^{\mathrm{XB}}$ & Pv02 & $2603-2626$ & SNP02_725 & SNP02_737 & E-PP8 ${ }^{\mathrm{XB}}$ & Pv08 & $110-170$ & SNP08_035 & SNP08_048 & 0.65 & 0.04 \\
\hline E-PP5 ${ }^{\mathrm{XB}}$ & Pv05 & 1191-1359 & 45 & SNP05_358 & E-PP11.2 $2^{\mathrm{XB}}$ & Pv11 & $1217-1387$ & $1 \_493$ & $1 \_518$ & 0.65 & 0.04 \\
\hline E-PA5.1 $1^{\mathrm{XB}}$ & Pv05 & $717-773$ & NP05_205 & SNP05_207 & E-PA7.1 ${ }^{\mathrm{XB}}$ & Pv07 & $214-261$ & SNP07_052 & SNP07_073 & -0.44 & 0.05 \\
\hline $\mathrm{PWI}^{\mathrm{XB}} *$ & Pv05 & $568-628$ & SNP05_106 & SNP05_108 & PWI1 $1^{\mathrm{XB} *}$ & Pv11 & $593-726$ & SNP11_186 & SNP11_202 & 0.01 & 0.01 \\
\hline $\mathrm{PL} 1.2^{\mathrm{XB} *}$ & Pv01 & $1336-1365$ & SNP01_543 & SNP01_562 & PL3.1 $1^{\mathrm{XB}} *$ & Pv03 & $657-724$ & SNP03_515 & SNP03_532 & -0.20 & 0.02 \\
\hline E-PL2 ${ }^{\mathrm{XB}}$ & Pv02 & $968-1090$ & SNP02_172 & SNP02_179 & E-PL3.2 $2^{\mathrm{XB}}$ & $\mathrm{Pv} 03$ & $1244-1392$ & SNP03_566 & SNP03_586 & 0.30 & 0.04 \\
\hline E-NSP1.1 ${ }^{\mathrm{XB}}$ & Pv01 & $374-422$ & SNP01_166 & SNP01_181 & E-NSP1 $11^{\mathrm{XB}}$ & Pv11 & $884-937$ & P11_240 & 1 1_285 & 0.18 & 0.07 \\
\hline
\end{tabular}

QTL named with the prefix E- only were detected in the two dimension genomes scan. E-QTL 1 and E-QTL 2 are the couple of QTL involved in the epistatic interaction. AA, estimated additive by additive epistatic effect in which positive values are provided by the parent Xana. $\mathrm{h}^{2}$, heritability of additive by additive effect; *, indicate that the QTL also is involved in epistatic interactions. (1) $\mathrm{p}$ value $<0.001$

Publisher's note Springer Nature remains neutral with regard to jurisdictional claims in published maps and institutional affiliations. 